\title{
Biological Relationships between miRNAs used for Colorectal Cancer Screening
}

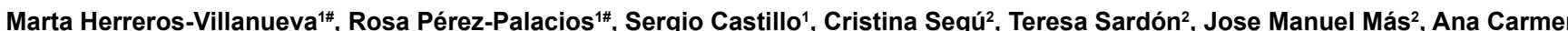
Martín ${ }^{1}$ and Rocio Arroyo ${ }^{*}$

${ }^{1}$ Advanced Marker Discovery (AMADIX), Acera De Recoletos, Valladolid, Spain

${ }^{2}$ Anaxomics Biotech, Balmes, Barcelona, Spain

\#Both authors contributed equally to this work

\begin{abstract}
MicroRNAs have been proposed to function as biomarkers in several disorders including cancer. Particularly, those present in plasma samples have been validated for colon cancer screening purposes. However, mechanisms underlying miRNA regulating CRC development have not been fully investigated yet. The aim of this study was to perform a biological contextualization of a particular 6 miRNAs signature (Hsa-miR-15b-5p, Hsa-miR-18a-5p, Hsa-miR-29a-3p, Hsa-miR335-5p, Hsa-miR-19a-3p and Hsa-miR-19b-3p) already described as CRC screening tool in terms of its downstream targets and associated pathways, to understand how this set of miRNAs could be regulating colon cancer development. Our results demonstrated that Hsa-miR-15b-5p, Hsa-miR-18a-5p and Hsa$\mathrm{miR}-29 \mathrm{a}-3 p$ are the best contributors to the diagnostic value of the signature by regulating epithelial to mesenchymal transformation though a gained balance of inhibiting tumoral suppressors genes such as ETS2, TP53, TLE3, ACVR2A.
\end{abstract}

Keywords: Biomarkers; Colorectal cancer; microRNA; Targets; Screening

Abbreviations: AA: Advanced Adenomas; ANNs: Neural Networks; CRC: Colorectal Cancer; EMT: Epithelial to Mesenchymal Transition; miRNA: microRNA; MTI: miRNA Target Interaction; PCR: Polymerase Chain Reaction; RT-qPCR: Quantitative Real Time Reverse Transcription PCR

\section{Introduction}

MicroRNAs (miRNAs) are a class of noncoding RNAs of about 22 nucleotides that mediate posttranscriptional gene expression mainly by either repressing the translation or inducing the degradation of their mRNA targets, to which they bind by complementarity [1].

Several authors have demonstrated that miRNAs are present in human circulation, plasma and serum, and profiles are altered in cancer $[2,3]$ being then suggested as a powerful new class of blood-based biomarkers.

Specifically, in CRC several signatures of miRNAs have been proposed to function as CRC screening biomarkers [4-6]. Advantages of miRNA used for CRC screening include stability due to miRNAs interaction with Argonaute 2, which keep it protected from nuclease activity [7]. Moreover, it is expected that blood-based test will increase adherence to screening programs because of its minimally invasive character. Although hot-topic in current research, indeed numerous authors have shown that miRNA are of interest for CRC diagnosis including miR-21, miR-29a, miR-92a, miR-532-3p, miR-331, miR-195, miR-17, miR-142-3p, miR-15b, miR-532, and miR-652, miR-409-3p, miR-7, and miR-93, miR-601 and miR-760, miR-23a-3p, miR-27a-3p, miR-142-5p, and miR-376c-3p, etc [8-10].

Previously we published a cohort of 196 individuals where patients with $\mathrm{CRC}$ and AA have significantly different patterns of miRNA expression than healthy individuals [5]. A set of miRNAs (Hsa-miR15b-5p, Hsa-miR-29a-3p, Hsa-miR-335-5p and Hsa-miR-18a-5p, HsamiR-19a-3p, Hsa-miR-19b-3p) demonstrated to have discriminative capacity when found in plasma samples. Then, in a case-control study of 300 subjects, a robust predictive model able to distinguish healthy individuals from those with advanced neoplasm (CRC or AA) was validated (data to be published).

Nevertheless, mechanisms underlying miRNA regulating CRC development have not been fully investigated yet. These studies are needed in order to construct robust panels that would be clinically validated. miRNA targets can be identified by prediction algorithms based on biophysical models, but further experimental strategies are essential to ensure that they are genuine $[11,12]$.

The aim of this study was to identify key proteins and pathways involved in how this particular 6 miRNA signature (Hsa-miR-15b-5p, Hsa-miR-18A-5p, Hsa-miR-29a-3p, Hsa-miR335-5p, Hsa-miR-19a-3p and Hsa-miR-19b-3p) already described as CRC screening tool could be regulating colon cancer development. A biological contextualization of the 6 miRNAs within colorectal carcinogenesis through a network-based approach was performed. By using systems biology based methods, these miRNAs were ranked based on the mechanistic relationship of their protein targets with CRC.

\section{Materials and Methods \\ miRNAs targets research}

The lists of gene targets from the 6 miRNAs signature were retrieved from miRTarBase $[13,14]$. Only experimentally validated targets were

${ }^{*}$ Corresponding author: Rocio Arroyo, Advanced Marker Discovery (AMADIX), Acera De Recoletos 2-1B, 47004, Valladolid, Spain, Tel: +034-9483-102060; E-mail: rarroyo@amadix.com

Received July 05, 2018; Accepted August 08, 2018; Published August 11, 2018

Citation: Villanueva MH, Palacios RP, Castillo S, Segú C, Sardón T, et al. (2018) Biological Relationships between miRNAs used for Colorectal Cancer Screening. J Mol Biomark Diagn 9: 398. doi: 10.4172/2155-9929.1000398

Copyright: @ 2018 Villanueva $\mathrm{MH}$, et al. This is an open-access article distributed under the terms of the Creative Commons Attribution License, which permits unrestricted use, distribution, and reproduction in any medium, provided the original author and source are credited. 
selected, in order to reduce the noise. Then, these genes were translated to proteins by mapping them to unique UniProt identifiers [15].

\section{Molecular characterization of colon cancer pathogenesis}

Colon cancer was characterized at molecular level via manual review of the literature. This characterization was performed in two steps: first identifying the main pathophysiological processes involved in CRC development and progression (referred to as physio pathological processes or motives), which were then further characterized at the protein level to provide a final list of 160 "effector" proteins (herein "effectors"). Hence, colon cancer 'effectors' are those proteins that, according to the existing literature, have previously been reported to play a critical role in this condition (databases: PubMed, ScienceDirect and Scopus).

\section{Generation of a protein map around colon cancer effectors}

"Effector" proteins were used to focus the analyses on the biological condition of interest in the human biological network. The direct interactions (physical interactions or functional relationships) between the miRNAs targets and the effectors of colon cancer, as well as the interactions among the protein targets, were assessed. Different publically available databases were consulted for the human protein network generation (e.g., Reactome, MINT, BioGrid) [16]. Cytoscape 3.5.1. software was used to perform the representation of all the reported interactions.

\section{Mechanistic evaluation of the miRNA target genes/proteins in the context of colon cancer: Artificial neural networks analysis}

The possible molecular relationship between the targets of miRNAs and colon cancer was evaluated by means of Artificial Neural Networks (ANNs), following TPMS technology protocols [17]. This approach implies the generation of mathematical models of the biological process through the use of artificial intelligence techniques. Then mathematical models were solved by ANNs, which are supervised algorithms that identify relations between the different nodes in the network. The system attempts to find the shortest distance between miRNA targets and the effectors of the disease or pathways, thus ranking the list of miRNA targets ordered by their association with the selected disease or pathway. ANNs analysis yields a predictive value ( $p$-value) that defines the probability of the existence of a relationship between each target and the diseases. Aiming to facilitate the understanding of the results, the obtained scores were divided in three categories: High $(\mathrm{p}<0,05)$; medium ( $\mathrm{p}<0.25)$; low $(\mathrm{p}>0.25)$. At this point, these individual target scores were aggregated into a cumulative value for each miRNA to allow their prioritization on a rational basis.

Relationships between the miRNAs targets and colon cancer were assessed both for individual proteins and for combinations of two proteins, to identify combinations of targets with a synergistic effect on colon cancer. The synergy criterion was applied according to the approach described by Berenbaum [18], in which a significant synergic effect is considered when the overall effect is $>20 \%$ of the sum of the individual effect of the two proteins. Additionally, the relationship between the miRNA targets and the different colon cancer motives (individually) was also calculated.

\section{Results}

\section{Identification of miRNAs targets}

According to the target profile overlap evaluation from miRTarBase-experimentally validated by other authors, the 6 miRNAs studied (Hsa-miR-15b-5p, Hsa-miR-18a-5p, Hsa-miR-29a-3p, HsamiR335-5p, Hsa-miR-19a-3p and Hsa-miR-19B-3p) seem to target different proteins, probably covering different pathophysiological mechanisms (Table 1).

When broad miRNA targets were investigated, Hsa-miR-335-5p is the miRNA presenting a higher proportion of unique targets compared to the rest of miRNAs. However, it should be noticed that Hsa-miR-335-5p is the one presenting the highest number of targets (2405 protein targets, 2 times more targets than the next miRNA, Hsa-miR-15b-5p with 1058). Hsa-miR-19a-3p and Hsa-miR-19b-3p are the miRNAs that present the lowest proportion of unique targets ( $2.8 \%$ and $18.4 \%$ respectively). In fact, their target profile overlaps substantially, probably because they belong to the miR-17-92 cluster located on chromosome 13q31.3 and could have potential redundant functions [19]. By contrast, Hsa-miR-18a-5p and Hsa-miR29a-3p had low proportion of targets, more than half of them being unique. Attending to the intensity of the reported miRNA target interaction (MTIs), Hsa-miR-29a-3p has a great proportion of MTIs identified through strong experimental techniques (72 strong and 112 weak interactions) (Table 1). Taking into consideration variability across miRNAs, it has to be noted that the proportion of shared targets over the total when considering duplicates $(36.6 \%)$ is clearly shorter than those unique to a single miRNA (63.4\%). When we examined these frequencies on all distinct protein miRNA targets, we observed that most targets are related to only one miRNA $(79.9 \%)$, whereas the rest (20.1\%) are silenced by two or more miRNAs.

\section{miRNA targets in CRC pathogenesis}

We then characterized CRC at molecular level by literature search (Table 2) and, based on these data, by using artificial neural networks we generated mathematical models of the disease (see Methods section). Through these models, we established a ranking of the identified unique or a combination of two miRNA targets according to their relationship to CRC and its pathophysiological processes (motives).

Our data demonstrated that the 6 miRNAs evaluated presented several proteins targets closely related to CRC showing different scores (Table 3). Results were rather homogenous, with around $70 \%$ of the

\begin{tabular}{|c|c|c|c|c|c|c|c|}
\hline miRNA & MTIs & Shared & $\%$ Shared & Unique & $\%$ Unique & Strong MTI & Weak MTI \\
\hline Hsa-mi-15b-5p & 1058 & 166 & $26.9 \%$ & 452 & $73.1 \%$ & 15 & 603 \\
\hline Hsa-mi-18a-5p & 292 & 89 & $40.8 \%$ & 129 & $59.2 \%$ & 21 & 197 \\
\hline Hsa-mi-29a-3p & 285 & 71 & $38.6 \%$ & 113 & $61.4 \%$ & 72 & 112 \\
\hline Hsa-mi-335-5p & 2405 & 196 & $10.4 \%$ & 1691 & $89.6 \%$ & 24 & 1863 \\
\hline Hsa-mi-19a-3p & 854 & 449 & $97.2 \%$ & 13 & $2.8 \%$ & 33 & 429 \\
\hline Hsa-mi-19b-3p & 1030 & 476 & $81.6 \%$ & 107 & $18.4 \%$ & 18 & 565 \\
\hline Total & 5924 & 1447 & $36.6 \%$ & 2505 & $63.4 \%$ & 183 & 3769 \\
\hline
\end{tabular}

Note: MTIs-miRNA Target Interactions; MTIs column represents the number of genes reported as miRNA targets.

Table 1: miRNA protein target identification. 
Citation: Villanueva MH, Palacios RP, Castillo S, Segú C, Sardón T, et al. (2018) Biological Relationships between miRNAs used for Colorectal Cancer Screening. J Mol Biomark Diagn 9: 398. doi: 10.4172/2155-9929.1000398

Page 3 of 6

total proteins showing low scores on average. However, there are some miRNAs that diverge from these values such as Hsa-miR-18a-5p and Hsa-miR-29a-3p.

The miRNAs presenting the highest proportion of targets highly related to CRC are Hsa-miR-18a-5p and Hsa-miR-29a-3p. Following them, we found Hsa-miR-19a-3p and Hsa-miR-19b-3p and HsamiR-15b-5p being Hsa-miR-335-5p the miRNA presenting the lowest proportion of targets highly related with CRC development. These data showed us that Hsa-miR-18a-5p and Hsa-miR-29a-3p are the miRNAs that binds with higher affinity to certain targets implicated in CRC development. Among the 6 miRNAs, Hsa-miR-29a-3p stands out as the candidate that displays better properties (high proportion of highly related) (more high and less low) as regulator of targets affecting colorectal carcinogenesis.

Looking at the 20 miRNA targets which display the best scores due to their relationship with CRC pathogenesis (Supplementary Table 1) we found proteins most usually described as oncogenes but also some tumor suppressor genes.

Among these 20 candidates we focused on Hsa-miR-15b-5p, Hsa-miR-18a-5p and Hsa-29a-3p as previously selected as the most important contributors for CRC pathogenesis (Table 4).

\begin{tabular}{|c|c|}
\hline Physio pathological process (motive) & $\begin{array}{c}\text { Number of Effector } \\
\text { Proteins }\end{array}$ \\
\hline $\begin{array}{c}\text { Tissue invasion and metastasis and Epithelial to } \\
\text { Mesenchymal Transition (EMT) }\end{array}$ & 71 \\
\hline Sustained angiogenesis & 27 \\
\hline Immune system suppression/resistance & 25 \\
\hline Cell growth and proliferation & 22 \\
\hline Inflammation & 17 \\
\hline Proteins related to epigenetic instability & 17 \\
\hline Evading apoptosis & 9 \\
\hline
\end{tabular}

Table 2: Summary of physiopathological pathways (motives) and effector proteins implicated in CRC development.
CRC contributors include both- oncogenes and tumoral suppressor proteins. In fact, a gained balance is obtained when looking to proteins acting as tumoral suppressors such as PDCD1, TET1, ETS2, TP53, TLE3, ACVR2A, TLE3. By contract, these 3 specific miRNAs could be targeting proteins that induce tumoral growth such as HIF1A, IGF1, GSK3b, VEGFA. These results show that altered expression of miRNAs is involved in deregulation of developmental programs and tumorigenesis. In CRC context, miRNA overexpression leads to an imbalance in tumoral suppressor genes and oncogenes haemostasis.

\section{miRNA targets by pathophysiological pathways (motive analysis)}

An analysis of the relationship of each miRNA protein target with CRC pathophysiological pathways was performed. According to these results, tissue invasion and metastasis- Epithelial to Mesenchymal transition (EMT) is assigned as the most relevant process (Table 5). In fact, EMT is the process that exhibits a higher number of miRNA targets with high association to effectors within CRC pathways (Supplementary Table 2). It has to be noted that process like immune system suppression/resistance and evading apoptosis possesses low levels of associations or weak functional relationship.

Table 5 shows that EMT is on average better represented within miRNA as opposed to evading apoptosis or inflammation. These data suggest that evaluated miRNAs may be related to CRC mainly through EMT. Results also demonstrate that Hsa-18a-5p and Hsa-miR29a$3 p$ are the two miRNAs more associated with CRC. On the contrary, values for Hsa-miR335-5p demonstrated its low importance probably due to the high number of targets reported for this miRNA.

\section{Data integration and final score for targets}

Targets combinations were also evaluated in order to identify combinations of proteins with effect on CRC. We found that when this effect was evaluated, results are similar than when evaluating individual targets: Hsa-miR-18a-5p and Hsa-miR29a-3p present

\begin{tabular}{|c|c|c|c|c|c|c|c|c|}
\hline miRNA & High & $\%$ High & Medium & $\%$ Medium & Low & $\%$ Low & Total & L/HM Ratio \\
\hline Hsa-mi-15b-5p & 11 & $1.8 \%$ & 163 & $26.4 \%$ & 444 & $71.8 \%$ & 618 & 2.6 \\
\hline Hsa-mi-18a-5p & 7 & $3.2 \%$ & 73 & $33.5 \%$ & 138 & $63.3 \%$ & 218 & 1.7 \\
\hline Hsa-mi-29a-3p & 7 & $2.8 \%$ & 63 & $34.2 \%$ & 114 & $62.0 \%$ & 184 & 1.6 \\
\hline Hsa-mi-335-5p & 30 & $1.6 \%$ & 384 & $20.3 \%$ & 1473 & $78.1 \%$ & 1887 & 3.6 \\
\hline Hsa-mi-19a-3p & 11 & $2.4 \%$ & 126 & $17.3 \%$ & 325 & $70.3 \%$ & 462 & 2.4 \\
\hline Hsa-mi-19b-3p & 12 & $2.1 \%$ & 151 & $25.9 \%$ & 420 & $72.0 \%$ & 583 & 2.6 \\
\hline Mean & 13 & $2.5 \%$ & 160 & $27.9 \%$ & 486 & $69.6 \%$ & 659 & 2.4 \\
\hline
\end{tabular}

Note: The last column contains the arithmetical ratio - low/(high+medium).

Table 3: miRNA target scores directly related to CRC pathogenesis. Percentage of affinity (high, medium or low) is represented.

\begin{tabular}{|c|c|c|c|}
\hline Protein name & Target Gene & Uni Prot_AC & miRNA ID \\
\hline Hypoxia-inducible factor 1-alpha & HIF1A & Q16665 & Hsa-miR-18a-5p \\
\hline Programmed cell death protein 1 & PDCD1 & Q15116 & Q8NFU7 \\
\hline Methyl cytosine dioxygenase TET1 & TET1 & P05019 & Hsa-miR-15b-5p \\
\hline Insulin-like growth factor I & IGF1 & Q15796 & Hsa-miR-29a-3p \\
\hline Mothers against decapentaplegic homolog 2 & SMAD2 & P15036 & Hsa-miR-18a-5p \\
\hline Protein C-ets-2 & ETS2 & P49841 & Hsa-miR-18a-5p \\
\hline Glycogen synthase kinase-3 beta & GSK3B & P15692 & Hsa-miR-335-5p, Hsa-miR-29a-3p, Hsa-miR-15b-5p \\
\hline Vascular endothelial growth factor A & VEGFA & P04637 & Hsa-miR-19b-3p, Hsa-miR-19a-3p, Hsa-miR-18a-5p \\
\hline Cellular tumor antigen $p 53$ & TP53 & Q04726 & Hsa-miR-18a-5p \\
\hline Transducin-like enhancer protein 3 & TLE3 & P27037 & Hsa-miR-15b-5p \\
\hline Activin receptor type-2A & ACVR2A & \\
\hline
\end{tabular}

Table 4: Best selection targets of Hsa-miR18a-3p, Hsa-miR15b-5p and Hsa-miR29a-3p by their relationship with CRC. 
the highest proportion of high-associated target pairs whereas HsamiR335-5p presents the highest proportion of low-associated target pairs. Importantly, Hsa-15b-5p presents the highest proportion of medium-associated target pairs but the low-associated targets pairs (even less than Hsa-miR-18a-5p and Hsa-miR-29a-3p) (Figure 1 and data not shown).

Finally, aiming to elaborate a final ranking that could enable effective prioritization of the miRNAs based on their targets, the results of previous analysis taking into account only high and medium relationship with CRC were integrated into a single ranking and a composite score was assigned accordingly. To that purpose, each parameter for each miRNA was assigned a value in a normalized score between 0 (worst) and 1 (best) and the average of single and combined was indicated as global score (Table 6). According to this final ranking, Hsa-miR-18a-5p, Hsa-miR29a-3p and Hsa-15b-5p are the miRNAs presenting highest scores of sensitivity in CRC targeting.

When we evaluated Hsa-miR-18a-5p, Hsa-miR29a-3p and Hsa$15 b-5 p$ showing high association with EMT and relationships between targets and motives we obtained a landscape composed by 25 effectors and 20 targets (Hsa-15b-5p having 13 targets, Hsa-miR29a-3p 1 targets and Hsa-miR-18a-5p 3 targets; 1 is shared between miR29a$3 p$ and Hsa-miR-18a-5p; 1 between Hsa-15b-5p and Hsa-miR-18a$5 p$ and 1 between Hsa-15b-5p and Hsa-miR29a-3p). Note worth that additionally 4 effectors-LAMC2, IGF1, ACVR2A, ETS2 are also targets simultaneously (Figure 2).

\section{Discussion}

In the present study, we performed a biological contextualization of a 6 miRNAs signature (Hsa-miR-15b-5p, Hsa-miR-29a-3p, HsamiR-335-5p and Hsa-miR-18a-5p, Hsa-miR-19a-3p, Hsa-miR19b-3p) previously described as biomarkers, within CRC screening setting. We elucidate which of these 6 miRNAs conferers the highest contribution to its diagnostic potential based on targets and effectors taking part in colorectal cancer development. We also provided evidence of functional cooperation between miRNA targets and EMT related effectors in CRC.

Our data highligthed that although 6 miRNAs included in the signature target proteins implicated in CRC development, Hsa-miR18a-5p, Hsa-miR29a-3p and Hsa-15b-5p are likely the most important contributors to the diagnostic value of the signature by regulating EMT process.

The signature evaluated in this study was originally identified on miRNAs overexpressed in AA and CRC tissues and further validated in plasma samples [5]. However, generally speaking- only a few miRNAs abundantly expressed in cancer cells are detectable in blood [20] and are those one with potential for early diagnosis purposes when minimally invasive methods are considered.
Hsa-miR-29a-3p was previously described as contributor for its diagnostic potential for high-risk adenomas [10]. In this study, miRNA29a together with miRNA92a in serum samples correlated with adenoma size and adenoma total number within the colorectum. Yang et al. [21] in a recent study have demonstrated that Hsa-miR-15b-5p are significantly associated with progression free survival (PFS) and/ or overall survival (OS) of colorectal cancer patients independent of tumor stage and age at diagnosis.

The miR-17-92 miRNA cluster generates a single polycistronic primary transcript that yields 6 mature miRNAs: miR-17, miR-18, miR-19a, miR-20a, miR-19b, and miR-22. Importantly, Hsa-miR-19a$3 p 23$ was identified in serum together with miR-21-5p and miR-425$5 p$ significantly higher in patients with CRC compared with controls and Hsa-miR-19b-3p was identified in a panel for the diagnosis of CRC [22].

Although these publications showed individual miRNAs as potential biomarkers for early diagnosis of AA and CRC, there is a need for more careful evaluation of targets and biological function that could support further clinical studies. A comprehensive literature research evidenced in our study indicated a functional role of miRNAs in colorectal carcinogenesis giving priority to targets and effectors driving CRC through EMT [23]

The more general targets the miRNA has, the less they seem to be associated to CRC development: while hsa-miR-29a-3p just covers 184 targets overall and presents a L/HM rate of 1,6, hsa-miR-335-5p covers 1887 targets (mapping for more high and medium targets, in absolute terms) and has the highest ratio $[3,6]$. This phenomenon could be caused by the fact that the targets of the miRNAs with less reported targets are better known proteins, which are better represented in the models, whereas some of the targets of hsa-miR-335-5p are less known proteins and are worse represented in the models. In fact, most of the MTIs reported for hsa-miR-335-5p (2299 out of 2405) have been identified in the same study [12], and a great proportion of its targets are reported through weak techniques [24].

Hsa-15b-5p has been associated to cell survival by affecting TP53 pathway through $\mathrm{Bcl} 2$ interaction in patients with inflammatory bowel diseases and associated cancer [25]. Also, this miRNA was found overexpressed in exosomes of colorectal cancer patients presenting shorter time to relapse for liver metastasis [26].

As commented previously, EMT has been extensively characterized in CRC while evading apoptosis, inflammation or epigenetic instability either has lower role in CRC or they are less studied. Attending to the two genes that seem to function as best targets as well as CRC effector with high scoring in EMT are ETS2 and ACVR2A. Both of them could be acting as promoters of carcinogenesis when inhibited by miRNAs. ETS2 gene encodes a transcription factor which regulates

\begin{tabular}{|c|c|c|c|c|c|c|}
\hline miRNA & $\begin{array}{c}\text { Cell growth and } \\
\text { proliferation }\end{array}$ & $\begin{array}{c}\text { Sustained } \\
\text { angiogenesis }\end{array}$ & $\begin{array}{c}\text { Evading } \\
\text { apoptosis }\end{array}$ & $\begin{array}{c}\text { Tissue invasion } \\
\text { and metastasis } \\
\text { (EMT) }\end{array}$ & $\begin{array}{c}\text { Immune system } \\
\text { Inflammation } \\
\text { suppression/ } \\
\text { resistance }\end{array}$ & $\begin{array}{c}\text { Proteins related } \\
\text { to epigenetic } \\
\text { instability }\end{array}$ \\
\hline Hsa-mi-15b-5p & 0.2 & 0.5 & 0.0 & 4.0 & 0.0 \\
\hline Hsa-mi-18a-5p & 2.3 & 0.5 & 0.0 & 8.3 & 0.0 \\
\hline Hsa-mi-29a-3p & 0.5 & 0.5 & 0.0 & 7.1 & 0.1 \\
\hline Hsa-mi-335-5p & 0.1 & 0.4 & 0.0 & 2.4 & 0.0 \\
\hline Hsa-mi-19a-3p & 1.3 & 0.2 & 0.0 & 6.5 & 0.1 \\
\hline Hsa-mi-19b-3p & 0.7 & 0.3 & 0.0 & 0.5 & 0.0 \\
\hline
\end{tabular}

Note: The percentage of targets that exhibits high relationship with a given process is showed. Green tones indicate high level of associated targets whereas red, orange and yellow correspond to a reduced association.

Table 5: Heatmap of miRNA targets with high association with each physio pathological process. 
Citation: Villanueva MH, Palacios RP, Castillo S, Segú C, Sardón T, et al. (2018) Biological Relationships between miRNAs used for Colorectal Cancer Screening. J Mol Biomark Diagn 9: 398. doi: 10.4172/2155-9929.1000398

Page 5 of 6

\begin{tabular}{|c|c|c|c|}
\hline miRNA & $\begin{array}{c}\text { Sensitivity Single } \\
\text { miRNA }\end{array}$ & $\begin{array}{c}\text { Sensitivity } \\
\text { Combined miRNAs }\end{array}$ & Global Score \\
\hline Hsa-miR-15b-5p & 0.39 & 1.00 & 0.67 \\
\hline Hsa-miR-18a-5p & 0.92 & 0.92 & 0.92 \\
\hline Hsa-miR29a-3p & 1.00 & 0.82 & 0.91 \\
\hline Hsa-miR335-5p & 0.00 & 0.00 & 0.00 \\
\hline Hsa-miR-19a-3p & 0.48 & 0.28 & 0.38 \\
\hline Hsa-miR19b-3p & 0.37 & 0.21 & 0.29 \\
\hline
\end{tabular}

genes involved in development and apoptosis. Its contribution may be specific to intestinal tumors driven by the Wnt pathway. Particularly, it has been described that ETS2 has an epithelial cell autonomous, tumor repressive activity for colon tumors [27]. ACVR2A encodes activin A type II receptor which belong to the transforming growth factor-beta (TGF-beta) superfamily of structurally related signalling proteins. In hypermutated tumours, ACVR2A is frequently mutated [28]. In fact, TGFBR2 and ACVR2A are commonly mutated in microsatellite unstable (MSI) colon cancers, which is the second most common

Table 6: Final ranking of miRNA biomarker candidates considering single targets and combinations. genomic subtype [29].

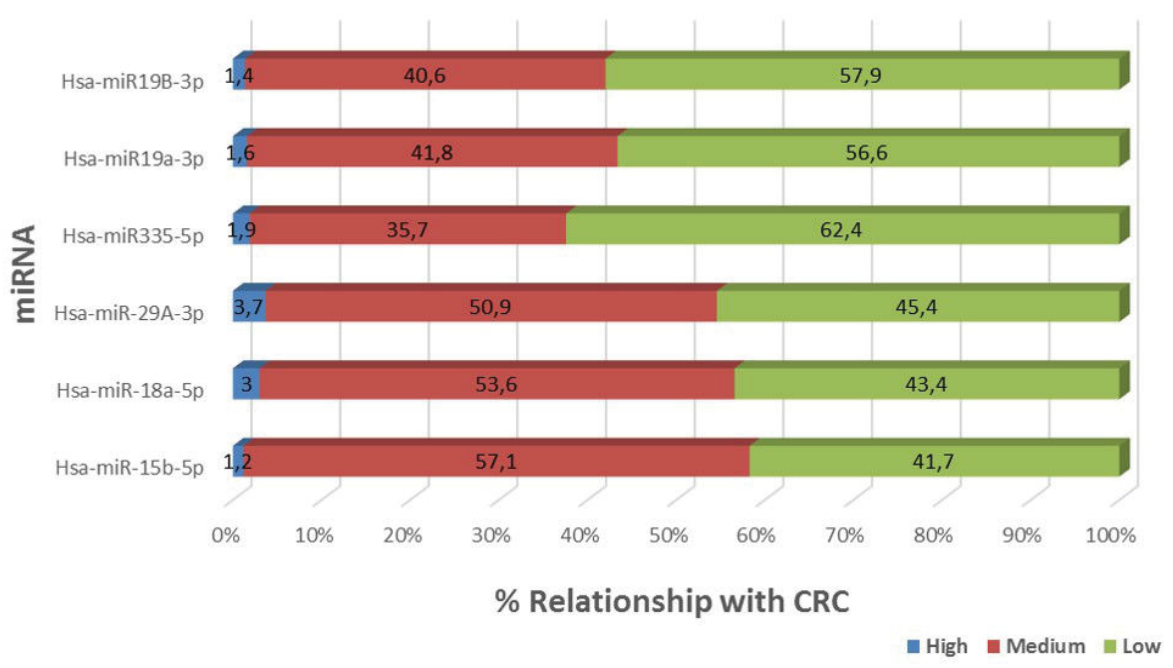

Figure 1: Representation of relationship of miRNA targets in pairs based on high, medium or low CRC association.

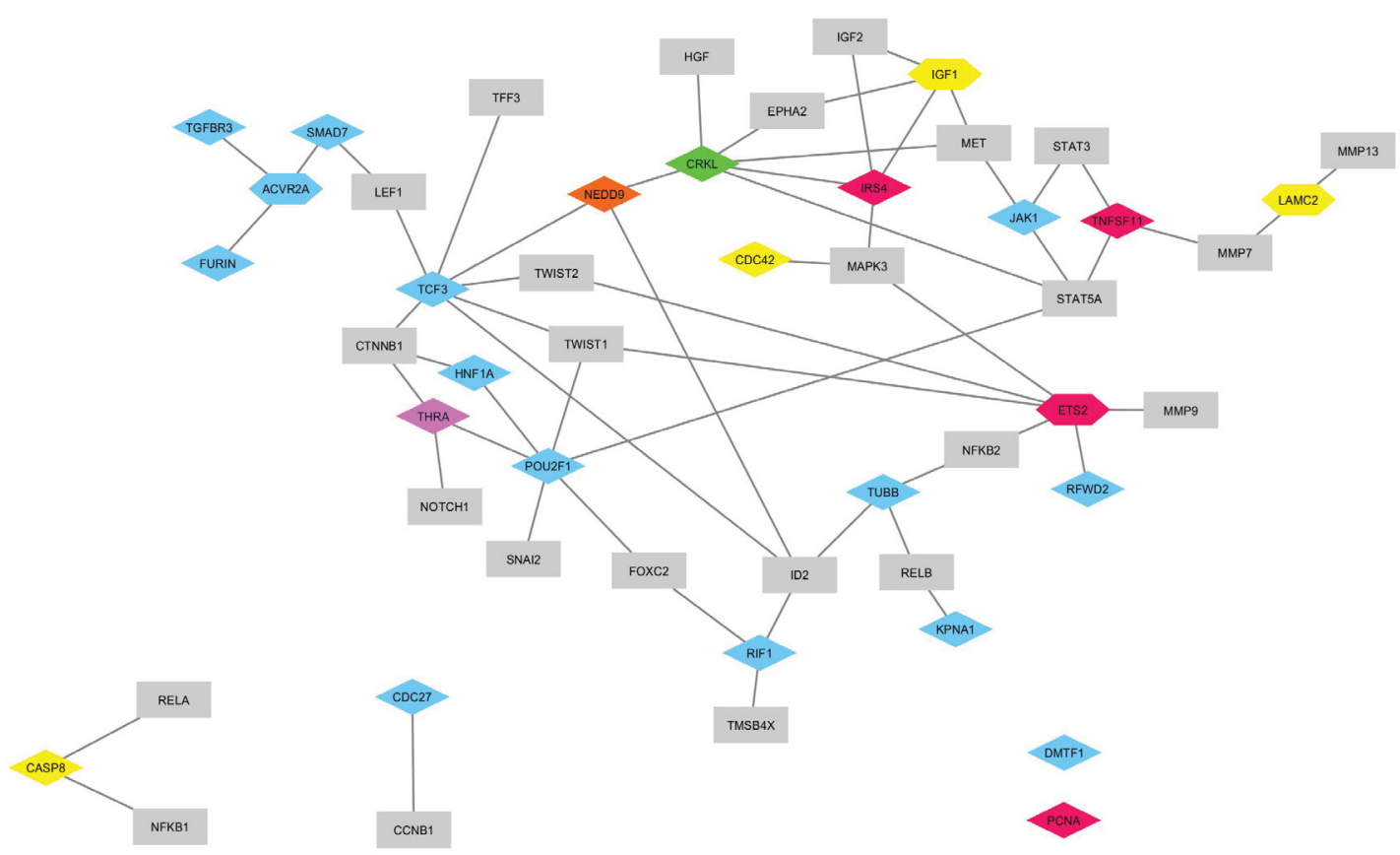

Figure 2: Network of EMT effectors and the targets of Hsa-miR-15b-5p, Hsa-miR-18a-5p and Hsa-miR29a-3p displaying a high score for this pysiophatological process, according to the mathematical models. Square: effector; Diamond: target; Hexagon: effector and target; Yellow: Hsa-miR29a-3p target; Blue: Hsa-miR15b-5p target; Red: Hsa-miR-18a-5p target. Orange: Hsa-miR-18a-5p and Hsa-miR29a-3p target; Pink: Hsa-miR-15b-5p and Hsa-miR-18a-5p target; Green: HsamiR-15b-5p a and Hsa-miR29a-3p target. Grey: No target. 
Citation: Villanueva MH, Palacios RP, Castillo S, Segú C, Sardón T, et al. (2018) Biological Relationships between miRNAs used for Colorectal Cancer Screening. J Mol Biomark Diagn 9: 398. doi: 10.4172/2155-9929.1000398

Surprisingly, LAMC2, IGF1 we also found as targets although have been traditionally described as overexpressed during cancer proliferation. IGF1 seems to be activated before carcinogenesis, and may promote the growth and malignant transformation of adenomatous polyps [30] and expression of laminin gamma 2 (LAMC2), matrix metallopeptidase 7 (MMP7) and EMT-related molecules were elevated in the colon tumors front [31]. Different interferences between other targets and effectors, as well as mutated, inactivated or inactivated genes could explain their implication here.

\section{Conclusion}

In summary, this study provides evidence for the potential targets of miRNAs and its usefulness of this signature for minimally invasive screening purposes. Hsa-miR-18a-5p, Hsa-miR29a-3p and Hsa-15b-5p are key regulators of CRC development mediating EMT, as proposed by different authors separately in previous studies.

Being a hot topic nowadays, miRNAs are a novel and specific type of biomarkers derived from tumor cells and its concentration is associated with invasive ability of the tumor cells and tumor microenvironment. Several publications hypothesized that certain miRNAs can be transferred to other cells and tissues free in plasma or by means of exosomes and build a suitable microenvironment for tumour growth and cell metastasis. Further studies are required in order to explore in detail biological function of miRNA and interactions in vivo.

\section{Acknowledgements}

Amadix team is very thankful to all board members.

\section{Grant support}

The present work was funded by Advanced Marker Discovery (AMADIX).

\section{Conflict of Interest}

Authors declare no conflict of interest.

\section{Author contributions}

M. Herreros-Villanueva, A. C. Martín, R. Pérez-Palacios, S. Castillo, designed the study and wrote the manuscript; Cristina Segú; Jose Manuel Más and Teresa Sardón performed the biocomputational analysis; R. Arroyo revised and approved the manuscript. All authors made comments and approved the manuscript.

\section{References}

1. Filipowicz W, Bhattacharyya SN, Sonenberg N (2008) Mechanisms of posttranscriptional regulation by microRNAs: Are the answers in sight? Nat Rev Genet 9: 102-114.

2. Chen X, Ba Y, Ma L, Cai X, Yin Y, et al. (2008) Characterization of microRNAs in serum: A novel class of biomarkers for diagnosis of cancer and other diseases. Cell Res 18: 997-1006.

3. Gilad S, Meiri E, Yogev Y, Benjamin S, Lebanony D, et al. (2008) Serum microRNAs are promising novel biomarkers. PLoS One 3: e3148.

4. Eslamizadeh S, Heidari M, Agah S, Faghihloo E, Ghazi H, et al. (2018) The role of microRNA signature as diagnostic biomarkers in different clinical stages of colorectal cancer. Cell J 20: 220-230.

5. Giráldez MD, Lozano JJ, Ramírez G, Hijona E, Bujanda L, et al. (2013) Circulating microRNAs as biomarkers of colorectal cancer: Results from a genome-wide profiling and validation study. Clin Gastroenterol Hepatol 11 681-688.

6. Stefanescu H, Muntean D, Pilut C, Diaconu M, Popescu R, et al. (2018) Using blood and plasma microRNAs as a non-invasive biomarker in patients with colorectal cancer. Clin Lab 64: 257-262.

7. Meister $\mathrm{G}$ (2013) Argonaute proteins: Functional insights and emerging roles. Nat Rev Genet 14: 447-459.

8. Wang S, Xiang J, Li Z, Lu S, Hu J, et al. (2015) A plasma microRNA panel for early detection of colorectal cancer. Int J Cancer 136: 152-161.
9. Vychytilova-Faltejskova P, Radova L, Sachlova M, Kosarova Z, Slaba K, et al. (2016) Serum-based microRNA signatures in early diagnosis and prognosis prediction of colon cancer. Carcinogenesis 37: 941-950.

10. Uratani R, Toiyama Y, Kitajima T, Kawamura M, Hiro J, et al. (2016) Diagnostic potential of cell-free and exosomal microRNAs in the identification of patients with high-risk colorectal adenomas. PLoS One 11: e0160722.

11. Thomson DW, Bracken CP, Goodall GJ (2011) Experimental strategies for microRNA target identification. Nucleic Acids Res 39: 6845-6853.

12. Hausser J, Zavolan M (2014) Identification and consequences of miRNAtarget interactions beyond repression of gene expression. Nat Rev Genet 15: 599-612.

13. Hsu JB, Chiu CM, Hsu SD, Huang WY, Chien $\mathrm{CH}$, et al. (2011) miRTar: An integrated system for identifying miRNA-target interactions in human. BMC Bioinformatics 12: 300 .

14. Chou CH, Chang NW, Shrestha S, Hsu SD, Lin YL, et al. (2016) miRTar base 2016: Updates to the experimentally validated miRNA-target interactions database. Nucleic Acids Res 44: D239-247.

15. Uni Prot C (2015) Uni Prot: A hub for protein information. Nucleic Acids Res 43: D204-212.

16. Herrando-Grabulosa M, Mulet R, Pujol A, Mas JM, Navarro X, et al. (2016) Novel neuroprotective multicomponent therapy for amyotrophic lateral sclerosis designed by networked systems. PLoS One 11: e0147626.

17. Fiuza-Luces C, Santos-Lozano A, Llavero F, Campo R, Nogales-Gadea G et al. (2018) Muscle molecular adaptations to endurance exercise training are conditioned by glycogen availability: A proteomics-based analysis in the McArdle mouse model. J Physiol 596: 1035-1061.

18. Berenbaum MC (1989) What is synergy? Pharmacol Rev 41: 93-141.

19. Ventura A, Young AG, Winslow MM, Lintault L, Meissner A, et al. (2008) Targeted deletion reveals essential and overlapping functions of the miR-17 through 92 family of miRNA clusters. Cell 132: 875-886.

20. Chen TS, Lai RC, Lee MM, Choo AB, Lee CN, et al. (2010) Mesenchymal stem cell secretes microparticles enriched in pre-microRNAs. Nucleic Acids Res 38: 215-224.

21. Yang J, Ma D, Fesler A, Zhai H, Leamniramit A, et al. (2017) Expression analysis of microRNA as prognostic biomarkers in colorectal cancer. Oncotarget 8: 52403-52412.

22. Tsuchida A, Ohno S, Wu W, Borjigin N, Fujita K, et al. (2011) miR-92 is a key oncogenic component of the miR-17-92 cluster in colon cancer. Cancer Sci 102: 2264-2271.

23. Zhu M, Huang Z, Zhu D, Zhou X, Shan X, et al. (2017) A panel of microRNA signature in serum for colorectal cancer diagnosis. Oncotarget 8: 17081-17091.

24. Zekri AR, Youssef AS, Lotfy MM, Gabr R, Ahmed OS, et al. (2016) Circulating serum miRNAs as diagnostic markers for colorectal cancer. PLoS One 11: e0154130.

25. Kanaan Z, Rai SN, Eichenberger MR, Barnes C, Dworkin AM, et al. (2012 Differential microRNA expression tracks neoplastic progression in inflammatory bowel disease-associated colorectal cancer. Hum Mutat 33: 551-560.

26. Monzo M, Santasusagna S, Moreno I, Martinez F, Hernández R, et al. (2017) Exosomal microRNAs isolated from plasma of mesenteric veins linked to live metastases in resected patients with colon cancer. Oncotarget 8: 30859-30869.

27. Múnera J, Ceceña G, Jedlicka P, Wankell M, Oshima RG (2011) Ets2 regulates colonic stem cells and sensitivity to tumorigenesis. Stem Cells 29: 430-439.

28. The Cancer Genome Atlas N (2012) Comprehensive molecular characterization of human colon and rectal cancer. Nature 487: 330-337.

29. Grady WM, Carethers JM (2008) Genomic and epigenetic instability in colorectal cancer pathogenesis. Gastroenterology 135: 1079-1099.

30. Zhang R, Xu GL, Li Y, He LJ, Chen LM, et al. (2013) The role of insulin-like growth factor 1 and its receptor in the formation and development of colorectal carcinoma. J Int Med Res 41: 1228-1235.

31. Kobayashi T, Masaki T, Nozaki E, Sugiyama M, Nagashima F, et al. (2015) Microarray analysis of gene expression at the tumor front of colon cancer Anticancer Res 35: 6577-6581. 